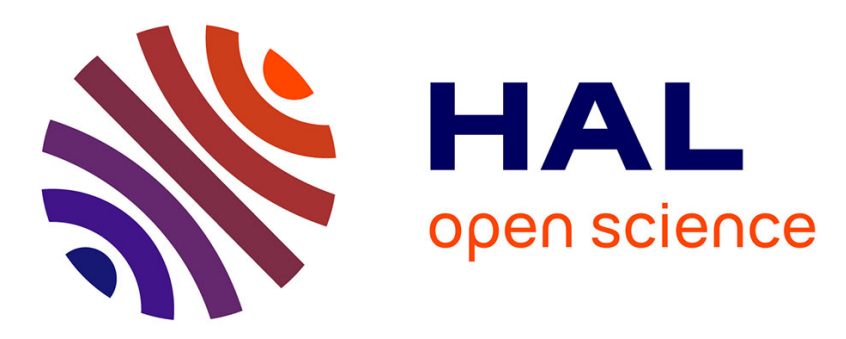

\title{
Magnetic particle chains embedded in elastic polymer matrix under pure transverse shear and energy conversion
}

Gildas Diguet, Gael Sebald, Masami Nakano, Mickaël Lallart, Jean-Yves Cavaillé

\section{To cite this version:}

Gildas Diguet, Gael Sebald, Masami Nakano, Mickaël Lallart, Jean-Yves Cavaillé. Magnetic particle chains embedded in elastic polymer matrix under pure transverse shear and energy conversion. Journal of Magnetism and Magnetic Materials, 2019, 481, pp.39-49. 10.1016/j.jmmm.2019.02.078 . hal02059863

\section{HAL Id: hal-02059863 https://hal.science/hal-02059863}

Submitted on 20 Oct 2020

HAL is a multi-disciplinary open access archive for the deposit and dissemination of scientific research documents, whether they are published or not. The documents may come from teaching and research institutions in France or abroad, or from public or private research centers.
L'archive ouverte pluridisciplinaire HAL, est destinée au dépôt et à la diffusion de documents scientifiques de niveau recherche, publiés ou non, émanant des établissements d'enseignement et de recherche français ou étrangers, des laboratoires publics ou privés. 
Magnetic particle chains embedded in elastic polymer matrix under pure transverse shear and energy conversion

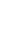

Gildas Diguet ${ }^{1}$, Gaël Sebald ${ }^{1}$, Masami Nakano ${ }^{1,2}$, Mickael Lallart $^{3}$, Jean-Yves Cavaillé ${ }^{1}$

${ }^{1}$ ELyTMaX UMI 3757, CNRS-Université de Lyon-Tohoku University International Joint Unit, Tohoku University, Sendai, Japan.

${ }^{2}$ New Industry Creation Hatchery Center, Tohoku University, Sendai, Japan.

${ }^{3}$ Univ. Lyon, INSA-Lyon, LGEF EA682, F69621 Villeurbanne, France.

\section{Abstract}

A model of structured Magneto-rheological Elastomer made of a soft matrix filled by magnetic particles was studied for energy harvesting, in pure shear condition. The particles were supposed to be aligned in a chain along which the magnetic field is applied and considering a shear strain applied in a plane perpendicularly to the chain. As the strain is applied and assuming the deformation is homogeneous and the particles remain aligned, both of the axis of their alignment and inter-distance between particles change, affecting their magnetic state. The aim of this work is to evaluate how far the magnetic induction variation induced by mechanical solicitation can be converted into electric signal. We firstly analyzed the magnetic susceptibility of a particle in the chain, and then derived an analytical expression for the composite permeability as a function of shear strain. FEM simulations taking into account the particle magnetic saturation was also performed to estimate the optimal conditions (magnetic field values, shear amplitude, etc.) maximizing the energy conversion potentials.

Keywords: Magneto-Rheological Elastomer, magnetization, pseudo-Villari, energy conversion.

\section{I- Introduction}

Magneto-Rheological Elastomers (MRE) consist of magnetically soft ferromagnetic particles embedded in an elastic polymer matrix. The strong magnetic behavior of the filler coupled with the highly deformable host, yields interesting behaviors in terms of magnetically tunable elastic properties and/or elastically tunable magnetic properties. A soft matrix filled by hard particles presents a larger Young modulus than the pure matrix (as, for instance, polydimethylsiloxane or PDMS), this reinforcement depends on the volume/weight fraction of particles (filling factor $\varphi$ ), their form factor (spherical, whiskers, platelets...) [1] and their spatial arrangement in the host matrix [2]. Moreover, if the particles are magnetic, the effective Young modulus is magnetically tunable and its increase is induced under magnetic field [2]. For instances, Varga et al., constructed anisotropic composites filled with carbonyl iron particles by applying a 400mT field during polymer curing for 5 hours. A second sample was cured without applying the magnetic field in order to get an isotropic composite filled by the same particles, for comparison purposes. It was shown through strain-stress measurements that the isotropic MRE exhibits a shear modulus $G_{i s o}(\varphi=30 \%, B=0)$ of $\sim 13 \mathrm{kPa}$ and for the anisotropic MRE, $G_{\text {aniso }}(\varphi=30 \%$, $B=0) \sim 55 \mathrm{kPa}$ for stress applied along the column of particles. Then, when exposed to a magnetic field during strain-stress tests, the isotropic MRE exhibited an increase of $G_{i s o}(\varphi=30 \%, B=0.1 \mathrm{~T})$ up to $15 \mathrm{kPa}$ whereas the anisotropic MRE showed $G_{\text {aniso }}(\varphi=30 \%, B=0.1 \mathrm{~T}) \sim 90 \mathrm{kPa}$ if the field is applied along the main axis of the aggregated particle segments while $G_{\text {aniso }}(\varphi=30 \%, B=0.1 \mathrm{~T}) \sim$ reaches only $60 \mathrm{kPa}$ if the field is applied perpendicularly to these segments [2]. This effect has received many attentions so far.

Another magneto-elastic coupling effect is the magnetically induced deformation of a MRE [3] which also depends on the applied field magnitude, filling factor, shape of the sample [4] and temperature [5], 
among other parameters. Furthermore, it has been observed that the measured deformation depends on the particle arrangement: for isotropic MRE, an extension [6,7] was observed along the applied field direction, and, on the contrary, a compression [7,8] was measured for anisotropic MRE, with magnetic particles arranged in column along the magnetic field axis.

These two examples of the elasto-magnetic effect have opened gates to possible applications such as magnetic damping $[9,10]$ and new actuator types $[11,12]$. Conversely, MRE can also be used as energy converting system to produce electricity $[13,14]$. In these articles, MRE samples were mounted in the gap of a magnetic circuit and were submitted to a shear strain. Shearing the sample modifies the magnetic induction which, in turn, induces an electric signal through a pick-up coil. Interestingly, the voltage produced is much larger in the case of anisotropic MRE than with an isotropic sample. A model assuming that the chain of particles is a single solid elongated magnetic particle which rotates to be aligned in the direction of the applied field has first proved the change of induction in the composite. However, in this model, one assumed no magnetic saturation of the particle.

We present here a model based on a single chain of spherical particles subjected to a shear strain and extract the change of magnetic induction as this chain is homogenously sheared. The aim of the present article is firstly to present the relationship between shear strain and magnetic behavior of the particles inside the chain. In this way, the magnetic susceptibility is expressed as a function of inter-particles distance, filling factor and strain. This model therefore helps to understand the magnetic properties and magnetic induction change of MRE under shear strain. Moreover, in a second step, the model includes the nonlinear magnetic behavior of the magnetic particles. As the local applied field is strong enough to reach the magnetic saturation of the particles, the magnetic properties of the particles are constant. That constant depends on the particle material.

The model therefore successfully allows predicting the quadratic behavior of the produced electrical signal and the existence of an optimal bias field, which is furthermore confirmed by experimental investigations.

\section{II-experiments}

A representation of the experimental device is given in Fig.1. An electric current $\left(i_{p}\right)$ is flowing through the primary coil (with $n_{p}$ turns), hence generating a magnetic field. This magnetic flux is then concentrated by the iron magnetic core (with magnetic permeability $\mu_{F e}$ ). The iron core has an air gap which is filled by two sheets of MRE (with an effective magnetic permeability $\mu_{M R E}$ ) and a sheet made of steel (with a magnetic permeability $\mu_{\text {steel }}$ ) in between them. The aim of the sheet is to apply the shear deformation $(\gamma)$ onto the two MRE sheets. MREs are such as their fillers are aligned perpendicularly to the steel sheet at rest $(\gamma=0)$, i.e. along the magnetic local field. Under a shear deformation, each particle magnetic state is modified. A change of magnetic induction therefore occurs which is then converted into an electric signal by the secondary coil (with $n_{s}$ turns) through Lenz law.

MREs used in the experiments were made of iron particles dispersed in a silicon rubber. Samples were labelled from their properties: typical shear modulus $G(\varphi, B=0)$ was around $200 \mathrm{kPa}$. Anisotropic MRE samples were obtained by applying a magnetic field $(0.3 \mathrm{~T})$ during the curing step of the rubber, whereas the isotropic sample was cured without such a field [13,14]. The two labels ISO and ANISO stands for isotropic and anisotropic MREs respectively. 


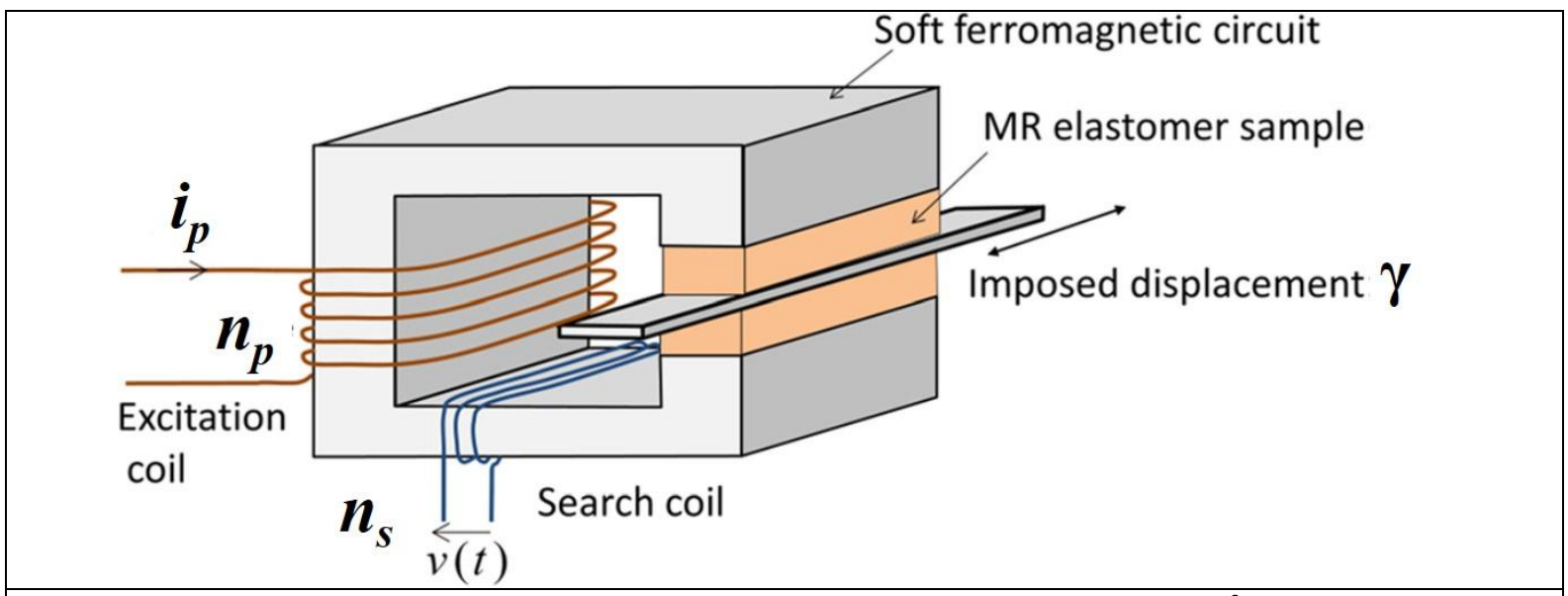

Fig.1 Experimental device. Soft ferromagnetic circuit cross section is $5 \times 1.2 \mathrm{~cm}^{2}$ and length $L_{F e}=20$ $\mathrm{cm}$, steel blade thickness $e_{s}=1 \mathrm{~mm}$, sample thickness $e=2 \mathrm{~mm}$, excitation coil winding number $n_{p}=1560$, search coil winding number $n_{s}=300[13,14]$.

1

From the flux conservation and Ampere's law, the equations that rule the magnetic system are obtained and a mathematical expression is found reckoning standard approximations [15]. First the length of the magnetic core is considered much longer than the air gap and, secondly, among the three permeabilities used here $\left(\mu_{F e}, \mu_{\text {steel }}\right.$ and $\left.\mu_{M R E}\right)$, we assume without loss of generality that $\mu_{M R E} \ll \mu_{F e}$, and $\mu_{F e} \sim \mu_{\text {steel }} \sim$ $10^{3}-10^{4} \mu_{0} ; \mu_{0}$ is the vacuum permeability with $\mu_{0}=4 \pi 10^{-7} \mathrm{H} / \mathrm{m}$. The flux density is finally approximated by:

$B \sim \frac{n_{p} i_{p} \mu_{M R E}}{2 e}=\frac{n_{p} i_{p} \mu_{0}}{2 e} \frac{\mu_{M R E}}{\mu_{0}}=\mu_{r_{-} M R E} B_{0}$

With $\mu_{r_{-} M R E}$ is the relative permeability of the $\operatorname{MRE}\left(\mu_{M R E}=\mu_{0} \mu_{r_{-} M R E}\right)$ and

$B_{0}=\mu_{0} \frac{n_{p} i_{p}}{2 e}=0.49 i_{p}$

where $B$ is the magnetic flux density flowing in the circuit $[15,16]$ and $B_{0}$ the induction created by an electromagnet without MRE in the effective gap of thickness $2 e$ (see Fig.1).

Two MRE samples were inserted in the magnetic circuit and the effective $B_{\text {meas }}$ was measured $[13,14]$ at different values of current $i_{p}$ without shear strain. The experiment was performed using anisotropic or isotropic MRE and corresponding results are presented in Fig.2a. For the sake of readability, we set the excitation field $H_{0}$ axis in Fig.2a using Eq.(2) and $H_{0}=B_{0} / \mu_{0}$. From Fig.2a, we can estimate the susceptibilities of each MRE. Guidelines in Fig.2a stand for relative susceptibility of 1 and the linear fit at low field (using the first two points and the origin as the intercept) of the two curves. At low applied field, the relative permeability $\mu_{r_{-} M R E}$ for the isotropic and anisotropic MRE are around 1.5 and 1.7 respectively. The anisotropy is clearly revealed here. Interestingly, at higher applied field $\left(H_{0} \geq 250\right.$ $\mathrm{kA} / \mathrm{m}$ ), the slopes are clearly close to 1 : it is therefore assumed that the magnetic particles got saturated and do not contribute anymore to the induction. By intersecting the low and high field linear behavior, it can be seen that the isotropic MRE is assumed to be saturated around $175 \mathrm{kA} / \mathrm{m}$ and the anisotropic MRE around $100 \mathrm{kA} / \mathrm{m}$. 

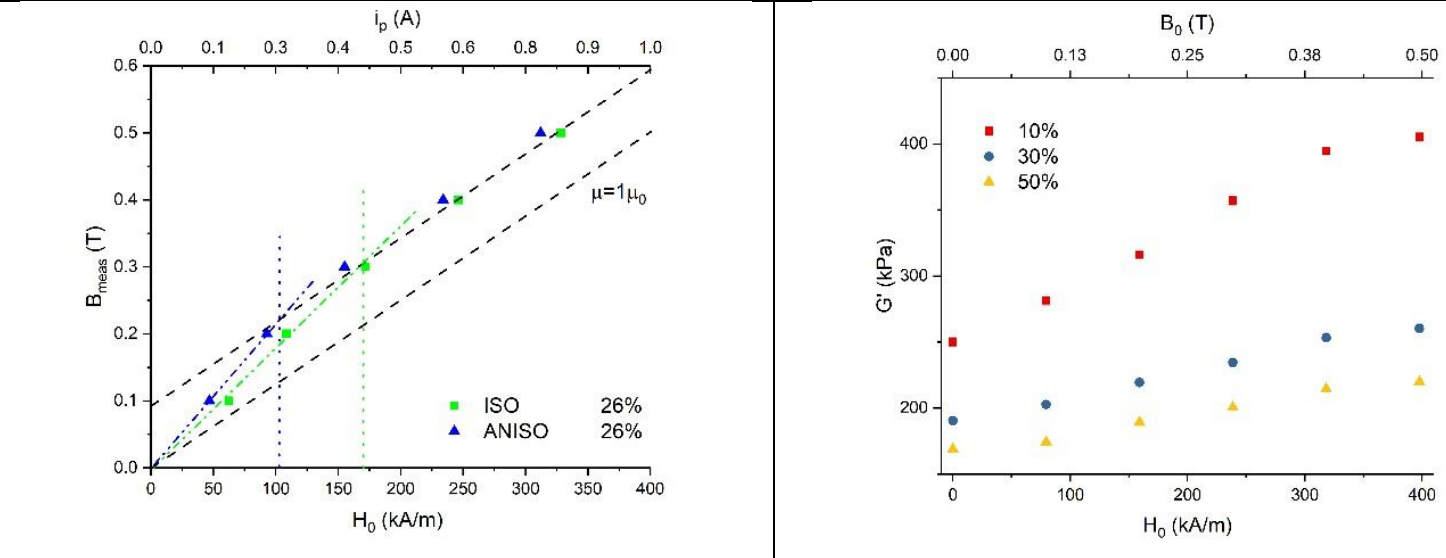

Fig.2. a). Effective magnetic flux measured close to the MRE. Dashed lines are guidelines for reader representing a relative permeability of 1 and 2. b). Elastic modulus as a function of the applied field for the anisotropic MRE. Experimental details are found in $[13,14]$

1

The strain and field impact on the anisotropic MRE mechanical properties is presented in Fig.2b, as the effective elastic modulus $G$ ' measured versus applied field. Similar to the zero-strain magnetic characteristic (Fig.2a), the curve presented two different behaviors at low and high fields respectively; at low field, below $250 \mathrm{kA} / \mathrm{m}$, a fast increase is observed whereas at high field, above $300 \mathrm{kA} / \mathrm{m}$, the increase is reduced. The separation between the two regions is relatively larger than the value found from the permeability curve.

In Fig.3, the induction change $\Delta B_{z}$ is plotted versus the applied strain for different external bias fields. The behavior of $\Delta B_{z}$ is an even function of the applied strain, originating from the even particle arrangement as a function of the strain. As the strain increases, a negative value of $\Delta B_{z}$ is observed, reckoning the applied field is non-null. The induction change $\Delta B_{z}$ is plotted versus the applied field too, and it can be seen that $\left|\Delta B_{z}\right|$ presents a maximal value in the range of $150-250 \mathrm{kA} / \mathrm{m}(0.2$ to $0.3 \mathrm{~T})$. Any further increase of applied field would reduce the effect on $\Delta B_{z}$.

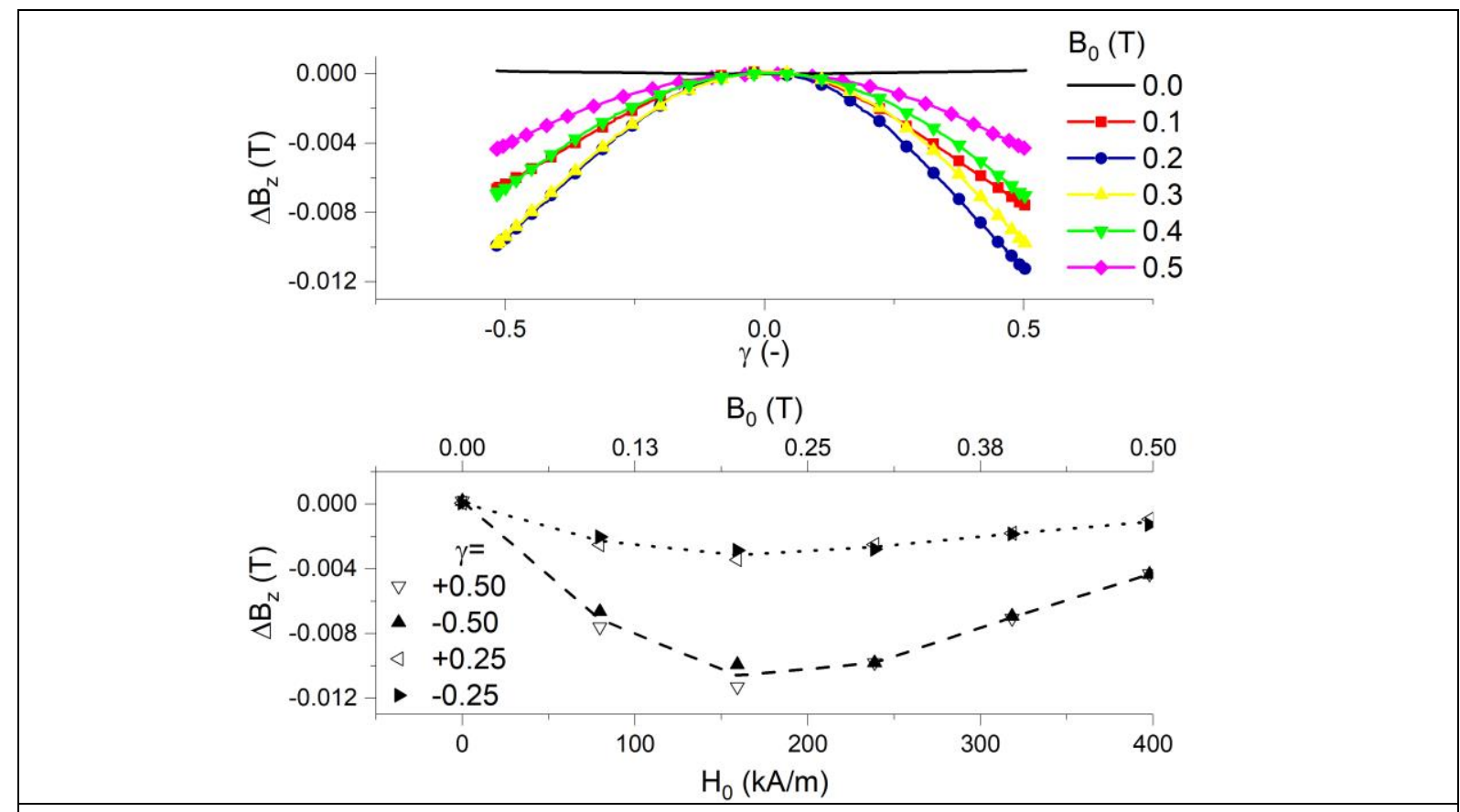

Fig.3. Effective magnetic induction change, measured at the secondary coil in anisotropic MRE Top: as a function of shear strain. Bottom: as a function of magnetic excitation at different strain. 
These Fig. 2 and Fig.3 highlight a change in the behavior in terms of susceptibility, magnetic flux and apparent Young modulus when the applied field is large enough. This can be interpreted as a consequence of particle magnetic saturation. In the following, we will model the chain with a linear magnetic behavior first (section III), for low field region, and then will use a FEM software (COMSOL) to account for the saturation threshold (section IV).

III- Linear magnetization of a particle chain

A magnetic spherical particle (with radius $a$ ) possesses a magnetic moment. It is by definition the product of the material magnetization by the particle volume:

$\vec{m}=\frac{4 \pi}{3} a^{3} \vec{M}_{p}$

$M_{p}$ is given as a function of (i) the local field $\boldsymbol{H}_{l o c}$ and (ii) permeability of the particle $\left(\mu_{p}\right)$ and of the matrix $\left(\mu_{m}\right)$ by:

$\vec{M}_{p}=3 \frac{\mu_{p}-\mu_{m}}{\mu_{p}+2 \mu_{m}} \vec{H}_{l o c}=3 \beta \vec{H}_{l o c}$

For a ferromagnetic particle embedded in a weak magnetic medium (usually $\mu_{\mathrm{m}} \sim \mu_{0}$ then $\mu_{\mathrm{p}} \gg \mu_{\mathrm{m}}$ ), Eq.(4) would be simplified into $\boldsymbol{M}_{p}=3 \boldsymbol{H}_{\text {loc }}(\beta \rightarrow 1)$, as discussed below. Because the local field $\boldsymbol{H}_{\text {loc }}$ is the one viewed by a particle, it results from the sum of the applied field $\boldsymbol{H}_{\boldsymbol{0}}$ and of the other particles field $\boldsymbol{H}_{\text {dip. }}$. The field $\boldsymbol{H}_{\text {dip }}$ is evaluated on a given particle as the sum of dipolar field $\boldsymbol{H}_{p}$ generated by each other particles magnetic moment. The local field $\boldsymbol{H}_{l o c}$ viewed by a test particle is then:

$\vec{H}_{l o c}=\vec{H}_{0}+\frac{1}{4 \pi} \sum_{i} \frac{\left(\vec{m}_{i} \cdot \frac{\vec{r}_{i}}{r_{i}}\right) \frac{\vec{r}_{i}}{r_{i}}-\vec{m}_{i}}{r_{i}^{3}}$

where the vector $\boldsymbol{r}_{i}$ is directed form a particle $i$, which carried a magnetic moment $\boldsymbol{m}_{i}$, to the test particle placed at $r=0$. In the following, we will assume the particles are all carrying the same moment, so the index will be dropped in the following.

Fig.4. A structure of chain of particles at different shear level; color map is the magnetic induction
in chain of particles. Applied magnetic field is directed along the z-axis.

The magnetic moment is supposed to have a component along the chain (subscript //) and a second one orthogonal to the chain (subscript $\perp$ ):

$\vec{m}=m_{/ /} \vec{u}_{/ /}+m_{\perp} \vec{u}_{\perp}$

The unit vectors $\vec{u}_{/ /}$and $\vec{u}_{\perp}$ are along and perpendicular to the chain respectively. 
1 If the other particles are regularly placed along the chain axis, with an inter-particle distance $d$

$2 \quad\left(\mathrm{~d}^{2}=\mathrm{d}_{\mathrm{y}}{ }^{2}+\mathrm{d}_{\mathrm{z}}{ }^{2}\right)$, the sum over $p$ particles is then modified into:

$3 \quad \sum_{r \neq 0} \frac{3\left(\vec{m} \cdot \frac{\vec{r}}{r}\right) \frac{\vec{r}}{r}-\vec{m}}{r^{3}}=\frac{2}{d^{3}} \sum_{p=1}^{\infty} \frac{3 m_{/ /} \vec{u}_{/ /}-m_{/ l} \vec{u}_{/ /}-m_{\perp} \vec{u}_{\perp}}{p^{3}}=\frac{4 m_{/ /} \vec{u}_{/ /}-2 m_{\perp} \vec{u}_{\perp}}{d^{3}} \sum_{p=1}^{\infty} \frac{1}{p^{3}}(7)$

4 Assuming chains are composed of many particles, the sum is converging to:

$5 \quad \sum_{p=1}^{\infty} \frac{1}{p^{3}}=S \approx 1.202$

6 From Eq.(3) to Eq.(8), we have:

$7 \quad\left(\begin{array}{l}H_{l o c, / l} \\ H_{l o c, \perp}\end{array}\right)=\left(\begin{array}{l}H_{0, / l} \\ H_{0, \perp}\end{array}\right)+\left(\begin{array}{c}4 S H_{l o c, / l} \\ -2 S H_{l o c, \perp}\end{array}\right) \beta \frac{a^{3}}{d^{3}}$

$8 \quad$ This local field can be rewritten to extract its relationship with the applied field.

$9 \quad\left(\begin{array}{l}H_{l o c, / /} \\ H_{l o c, \perp}\end{array}\right)=\left(\begin{array}{c}\frac{\cos (\theta)}{1-\beta 4 S \frac{a^{3}}{d^{3}}} \\ \frac{\sin (\theta)}{1+\beta 2 S \frac{a^{3}}{d^{3}}}\end{array}\right) H_{0}$

12

where $\theta$ is the angle between the chain direction and the applied field direction. This allows expressing the particle magnetization, Eq.(4), as:

$$
\vec{M}_{p}=\frac{3 \beta \cos (\theta) H_{0}}{1-\beta 4 S \frac{a^{3}}{d^{3}}} \vec{u}_{/ /}+\frac{3 \beta \sin (\theta) H_{0}}{1+\beta 2 S \frac{a^{3}}{d^{3}}} \vec{u}_{\perp}
$$

Last step in the calculation is to project this magnetization into a coordinate system defined along the applied field axis $\left(u_{z}\right)$ and perpendicularly to it $\left(u_{y}\right)$.

$$
M_{p z}=\vec{M}_{p} \cdot \vec{u}_{z}=\frac{3 \beta H_{0}}{1-\beta 4 S \frac{a^{3}}{d^{3}}}-\sin ^{2}(\theta)\left[\frac{3 \beta H_{0}}{1-\beta 4 S \frac{a^{3}}{d^{3}}}-\frac{3 \beta H_{0}}{1+\beta 2 S \frac{a^{3}}{d^{3}}}\right]
$$

and

$M_{p y}=\vec{M}_{p} \cdot \vec{u}_{y}=\cos (\theta) \sin (\theta)\left[\frac{3 \beta H_{0}}{1-\beta 4 S \frac{a^{3}}{d^{3}}}-\frac{3 \beta H_{0}}{1+\beta 2 S \frac{a^{3}}{d^{3}}}\right]$

Dividing these expressions by the applied field value $H_{0}$ yields to the apparent susceptibility of a particle in the chain as function of the angle between the chain orientation and the applied field. In other words, this is the pure rotation-induced apparent susceptibility of particle in the chain. It is expressed along the field axis $\left(\chi_{z_{-} a}\right)$ and transverse to the field $\left(\chi_{y_{-} a}\right)$ : 
1

$\chi_{z_{-} a}=\frac{3 \beta}{1-\beta 4 S \frac{a^{3}}{d^{3}}}-\sin ^{2}(\theta)\left[\frac{3 \beta}{1-\beta 4 S \frac{a^{3}}{d^{3}}}-\frac{3 \beta}{1+\beta 2 S \frac{a^{3}}{d^{3}}}\right]$

$\chi_{y_{-} a}=\cos (\theta) \sin (\theta)\left[\frac{3 \beta}{1-\beta 4 S \frac{a^{3}}{d^{3}}}-\frac{3 \beta}{1+\beta 2 S \frac{a^{3}}{d^{3}}}\right]$

3 Eq. (14) shows that $\chi_{z_{-} a}\left(\theta=0^{\circ}\right)=3 \beta /\left[1-4 \beta S(a / d)^{3}\right]$ and $\chi_{z_{-} a}\left(\theta=90^{\circ}\right)=3 \beta /\left[1+2 \beta S(a / d)^{3}\right]$ and generally, 4 susceptibilities can be rewritten as $\chi_{z \_a}(\theta)=\chi_{z_{z} a}\left(\theta=0^{\circ}\right)-\sin ^{2}(\theta)\left[\chi_{z \_a}\left(\theta=0^{\circ}\right)-\chi_{z \_a}\left(\theta=90^{\circ}\right)\right]$. Similarly, Eq. (15) 5 can be rewritten as $\chi_{y_{\_} a}(\theta)=\cos (\theta) \sin (\theta)\left[\chi_{z_{-} a}\left(\theta=0^{\circ}\right)-\chi_{z_{-} a}\left(\theta=90^{\circ}\right)\right]$. The term $\left[\chi_{z_{-} a}\left(\theta=0^{\circ}\right)-\chi_{z_{-} a}\left(\theta=90^{\circ}\right)\right]$ is 6 then important to characterize the anisotropic behavior of the chain: if it is null, the material is isotropic 7 and only $\chi_{z \_a}$ remains, i.e. the magnetization is directed along the applied field.

8 This description corresponds to the pure rotation of the chain. But to account for the shear effect, another 9 contribution must be introduced. In the following, a chain with an initial orientation along $\boldsymbol{u}_{z}$ is submitted 10 to a shear strain along $\boldsymbol{u}_{\boldsymbol{y}}$.

11 The shear strain $\gamma$ is defined as:

$\gamma=\frac{d_{y}}{d_{z}}=\tan (\theta)$

During the pure shear, only the distance $d_{y}$ is changing while $d_{z}$ remains constant $\left(d_{z}=d_{0}\right)$. Setting a chain structure constant as $2 D=4 \mathrm{~S}\left(\mathrm{a} / \mathrm{d}_{0}\right)^{3}$ and using formula exposed in Appendix A, the two components of the susceptibility becomes:

$\chi_{z_{-} a}=\frac{3 \beta}{1-\beta 2 D \frac{1}{\left(\gamma^{2}+1\right)^{3 / 2}}}-\frac{\gamma^{2}}{\gamma^{2}+1}\left[\frac{3 \beta}{1-\beta 2 D \frac{1}{\left(\gamma^{2}+1\right)^{3 / 2}}}-\frac{3 \beta}{1+\beta D \frac{1}{\left(\gamma^{2}+1\right)^{3 / 2}}}\right]$

$\chi_{y_{-} a}=\frac{\gamma}{\gamma^{2}+1}\left[\frac{3 \beta}{1-\beta 2 D \frac{1}{\left(\gamma^{2}+1\right)^{3 / 2}}}-\frac{3 \beta}{1+\beta D \frac{1}{\left(\gamma^{2}+1\right)^{3 / 2}}}\right]$

The z-axis susceptibility can then be rewritten as:

$$
\chi_{z_{-} a}=\alpha(\gamma, 1)-\frac{\gamma^{2}}{\gamma^{2}+1}[\alpha(\gamma, 1)-\alpha(\gamma,-1 / 2)]
$$

where

$$
\alpha(\gamma, k)=\frac{3 \beta}{1-k \beta 2 D \frac{1}{\left(\gamma^{2}+1\right)^{3 / 2}}}
$$


The apparent susceptibility as a function of the angle of rotation (for the pure rotation), and as a function of the shear angle (for the pure shear) at different initial $\mathrm{d}_{\mathrm{z}} / 2$ a ratio is plotted in Fig.5, along with FEM analysis results for comparison purposes.

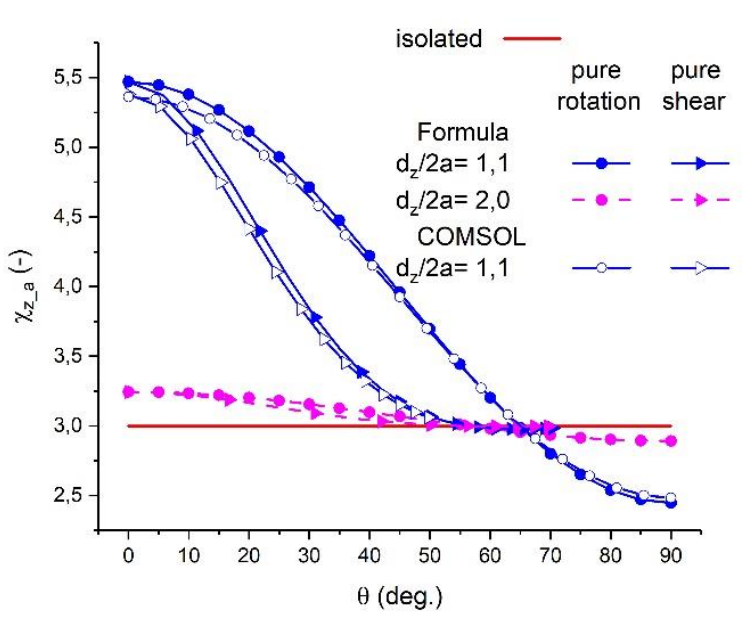

Fig.5. Apparent susceptibility of a single particle in a chain (line with markers) as a function of the angle of rotation (for the pure rotation case) and of the shear angle (for the pure shear case) for two different initial $\mathrm{d}_{z} / 2$ a ratios and comparison with apparent susceptibility of an isolated particle (red line without markers)

5

For an isolated particle placed in a non-magnetic medium $(\beta=1)$ where a magnetic field $\boldsymbol{H}_{\boldsymbol{0}}$ is applied, the particle magnetization $\boldsymbol{M}_{p}$ at low field is $\boldsymbol{M}_{p}=3 \boldsymbol{H}_{\boldsymbol{0}}$, turning into an apparent susceptibility $\chi_{z_{\_} \mathrm{a}}=3$. In the case of the chain of particles being purely rotated, if the chain and the applied field $\boldsymbol{H}_{\boldsymbol{0}}$ are parallel $\left(\theta=0^{\circ}\right)$, the field created by the other particles fields $\boldsymbol{H}_{d i p}$ is enhancing $\boldsymbol{H}_{\boldsymbol{0}}$ and the particle magnetization is increased whereas if the chain and the applied field $\boldsymbol{H}_{\boldsymbol{0}}$ are perpendicular $\left(\theta=90^{\circ}\right)$, the particle magnetization is decreased because $\boldsymbol{H}_{d i p}$ is lowering $\boldsymbol{H}_{\boldsymbol{0}}$. The variation depends on the $d_{z} / 2 a$ ratio; for $d_{z} / 2 a=1.1$, the apparent susceptibility is reduced from $\chi_{z_{\_} a}\left(\theta=0^{\circ}\right)=5.5$ to $\chi_{z_{-} a}\left(\theta=90^{\circ}\right)=2.5$ while this variation of apparent susceptibility is smaller for $d_{z} / 2 a=2.0$. In the case of a pure shear process, the inter-particle distance increases with shear increase, which contributes to further reducing the apparent susceptibility.

In both cases, i.e. simple rotation or pure shear, the initial $d_{z} / 2 a$ ratio is tuning the field reinforcement. The main difference between these two cases is the fact that the distance is constant for pure rotation unlike to the case of pure shear. This means that pure shear tends to demagnetize the particles $\left(\chi_{z_{\_} a}<3\right)$ in a smaller chain angle when compared to pure rotation.

Up to now, calculation considered a single particle property. In the following, the susceptibility of a model composite material, made of one chain of these particles embedded in a non-magnetic matrix is evaluated. To accomplish this calculation, the homogenization procedure would need to take into account the "cavity field" into the local field [17]. This cavity field is usually expressed as being proportional to $1 / 3$ of the effective composite magnetization, i.e. $\boldsymbol{H}_{c a v}=\boldsymbol{M}_{c} / 3$. The composite magnetization is $\boldsymbol{M}_{\boldsymbol{c}}=\phi \boldsymbol{M}_{\boldsymbol{p}}$ where $\phi$ is the volume filling factor (volume of all the particles divided by the volume of the composite). The susceptibility of the composite under pure shear is then: 
1

$\chi_{z}=\frac{3 \phi \beta}{1-\beta(\phi+2 D)}-\frac{\gamma^{2}}{\gamma^{2}+1}\left[\frac{3 \phi \beta}{1-\beta\left(\phi+2 D \frac{1}{\left(\gamma^{2}+1\right)^{3 / 2}}\right)}-\frac{3 \phi \beta}{1-\beta\left(\phi-D \frac{1}{\left(\gamma^{2}+1\right)^{3 / 2}}\right)}\right]$

For a composite material, without applied strain $(\gamma=0)$, the magnetization is as $\boldsymbol{M}=3 \beta \phi /[1-\beta(\phi+2 D)] \boldsymbol{H}_{\boldsymbol{0}}$ where $D$ is a term corresponding to a given architecture. It is noteworthy that this expression is similar to the expression of the composite susceptibility given by Martin [17], $\boldsymbol{M}=3 \beta \phi /\left[1-\beta\left(\phi-2 \Psi_{z}\right)\right] \boldsymbol{H}_{0}$ : the numerical parameter $\Psi_{z}$ that takes into account the effect of all particles is negative for a column placed in the direction of the applied field. On the other hand, by taking the limit of $\beta=1$ (highly permeable particle in low permeable medium), and in the case of randomly-filled composite, the well-known Maxwell-Garnett equation (for random distribution) is written as $\boldsymbol{M}=3 \phi /[1-\phi] \boldsymbol{H}_{\boldsymbol{o}}$ : it is easy to obtain it using the previously exposed expression by setting $D \sim 0$ (the average distance between particles is much larger than their radius, i.e. $d_{0}>>a$ ).

In order to account for the variation of permeability induced by the application of a shear strain on the model composite, the magnetic susceptibility of the composite is injected into the permeability expression as:

$\mu_{z}=\mu_{0}\left(1+\chi_{z}\right)$

where $\chi_{z}$ is given by Eq.(21).

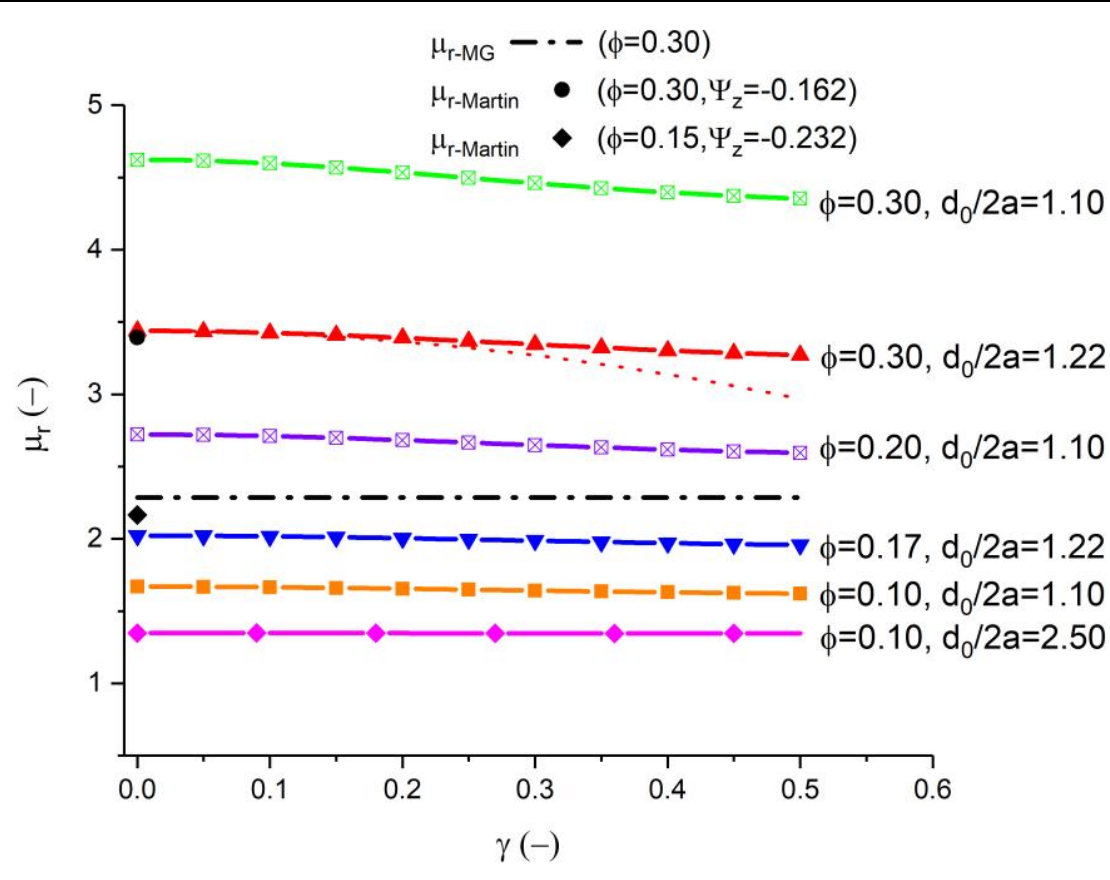

Fig.6. Relative permeabilities versus shear strain for composite with different filling factor and inter-particle distance, calculated with Eq.(21) and (22) and compared with Martin et al. value [17] and Maxwell-Garnett (MG) value, respectively. The dotted line corresponds to the Taylor expansion of the permeability (see text for details)

Composite relative permeabilities $\left(\mu_{r}=\mu_{z} / \mu_{0}\right)$ are calculated using Eq.(21) and Eq.(22). Resulting curves are presented in Fig.6. Without applied shear strain $(\gamma=0)$ and with the same filling factor, the effect of the inter-particle distance on the effective permeability obtained with Eq.(21)-(22) is shown; for example, at $\phi=0.3$, the largest permeability $\mu_{r}=4.62$ is obtained with $d_{z} / 2 a=1.10$, while for $d_{z} / 2 a$ 
$=1.22$, the permeability value is then $\mu_{r}=3.44$, which is close to the value found by Martin equation for a composite with $\phi=0.3$ magnetized along the chain direction. Interestingly, the randomly-filled composite with $\phi=0.3$ is $\mu_{r}=2.3$ according to the Maxwell-Garnett formula as seen in Fig.6. The anisotropic susceptibilities calculated by Martin [17] and our model are larger than the isotropic susceptibilities. Similarly, as the filling factor increases (i.e., increasing the quantity of magnetic materials) the permeability increases: with $d_{z} / 2 a=1.10, \mu_{r}=1.67$ for $\phi=0.1$ and $\mu_{r}=4.62$ for $\phi=0.3$. However, it is important to remember that this model is based on a single chain of particles, which takes into account interaction only from particles in the chain. Adding more particles in a single chain weakly change the magnetic behavior since the sum $S$ (Eq.8) is rapidly convergent. More realistically, at large filling factor, they would be many chains and it is highly probable that particles interact not only with each other within a chain, but also with particles from neighbor chains too. Boczkowska measured anisotropic MRE susceptibilities perpendicularly and parallel to the chain direction or for different filling factor [18]. He showed experimentally that the two components of the susceptibility are almost equals for a composite filled by $33 \%$ (vol.). In these composites, image analysis [19] reveals that the mean distance between chains were nearly equal to the inter-particle distance within chain. Therefore, such composites, above $33 \%$ of fillers, are no more anisotropic, and factor $D$ should be modified to account for the filling factor. This was numerically done in the data provided by Martin [17]. In this way, our analytical model with a $D$ independent from the filling factor is tending to overestimate susceptibility values as the filling factor becomes closer to the $33 \%$ value. However, our model can analytically describe the physical effect of the strain on the susceptibility and this is rarely provided in the literature.

The general behavior of the permeability with strain, which is similar to the susceptibility of a single particle in a sheared chain (Fig.5), can be divided into three zones: as an example, in Fig.6 with $\phi=0.3$ and $d_{z} / 2 a=1.10$, an initial quadratic curve for strain ranging from $\gamma=0$ to 0.15 is followed by a linear part between $\gamma=0.15$ to 0.40 and finally a smoothing of the curve for $\gamma>0.40$. This behavior is less obvious for different composite parameters. When the strain is applied, the particles are moving away from each-others, so that the distance between particle is increasing and the angle between their magnetization and chain direction is also increasing from the initial angle $\theta=0^{\circ}$. The combination of these two effects induces the local field reduction, as discussed in the previous section, and then the particle magnetization is reduced too. It is important to notice that the change of permeability is larger when the gap between neighboring particles is small.

Some calculated values of relative permeability are extracted from Fig. 6 and presented in Table 1. As the filling factor increases the composite permeability increases; e.g., with $d_{z} / 2 a=1.10$, relative permeabilities are 1.67 at $\phi=0.1,2.72$ at $\phi=0.2$ and 4.62 at $\phi=0.3$. Experimental data extracted from Fig. 3 are presented in Table 1 too.

Table1. Relative permeabilities for different initial inter-particle distances and filling factors. Values are given without applied strain $\mu_{r}(\gamma=0)$ and with a strain $\mu_{r}(\gamma \neq 0)$; changes of permeability $\Delta \mu_{r}(\gamma \neq 0)=$ $\mu_{r}(\gamma=0)-\mu_{r}(\gamma \neq 0)$ are also provided.

\begin{tabular}{|c|c|c|c|c|c|c|c|}
\hline $\begin{array}{c}\text { Extracted } \\
\text { from }\end{array}$ & $d_{0} / 2 a$ & $\phi$ & $\mu_{r}(\gamma=0)$ & $\mu_{r}(\gamma=0.25)$ & $\mu_{r}(\gamma=0.50)$ & $\begin{array}{c}\mu_{r}(\gamma=0.25) \\
-\mu_{r}(\gamma=0)\end{array}$ & $\begin{array}{c}\mu_{r}(\gamma=0.50) \\
-\mu_{r}(\gamma=0)\end{array}$ \\
\hline Fig.6 & 1.10 & 0.10 & 1.67 & 1.65 & 1.62 & -0.02 & -0.05 \\
\hline Fig.6 & 1.10 & 0.20 & 2.72 & 2.67 & 2.60 & -0.06 & -0.13 \\
\hline Fig.6 & 1.10 & 0.30 & 4.62 & 4.50 & 4.35 & -0.13 & -0.27 \\
\hline Fig.6 & 1.22 & 0.17 & 2.02 & 2.00 & 1.96 & -0.03 & -0.06 \\
\hline Fig.6 & 2.50 & 0.10 & 1.35 & 1.35 & 1.35 & -0.00 & -0.00 \\
\hline Fig.3 & \multicolumn{7}{|r}{} \\
\hline
\end{tabular}


As the susceptibility obtained through Eq.(21) and (22) can be difficult to interpret as the shear adds complexity in the formula, a more easily interpretable formula can be obtained using a Taylor-Young expansion around $\gamma=0$ for low shear. This expansion at low shear strain is:

$\mu_{z}(\gamma=0) \approx \mu_{z}(\gamma=0)+\frac{\partial \mu_{z}(\gamma=0)}{\partial \gamma} \gamma+\frac{1}{2} \frac{\partial^{2} \mu_{z}(\gamma=0)}{\partial \gamma^{2}} \gamma^{2}+O\left(\gamma^{3}\right)$

By using formula exposed in Appendix B, we obtain:

$\mu_{z}(\gamma=0) \approx \mu_{0}\left[1+\frac{3 \phi \beta}{1-\beta(\phi+2 D)}-\left(D \frac{9 \phi \beta^{2}}{(1-\beta(\phi+2 D))^{2}}+\left[\frac{3 \phi \beta}{1-\beta(\phi+2 D)}-\frac{3 \phi \beta}{1-\beta(\phi-D)}\right]\right) \gamma^{2}\right]$

The magnetic susceptibility dependence with shear strain is then rewritten as:

$\mu_{z}(\gamma \approx 0) \approx \mu_{0}\left(1+\frac{3 \phi \beta}{1-\beta(\phi+2 D)}-b \gamma^{2}\right)=\mu_{z}(\gamma=0)-\mu_{0} b \gamma^{2}$

where $b$ is a constant obtained by identification of Eq.(24) and Eq.(25).

In the case of a composite with $d_{z} / 2 a=1.22$ and $\phi=30$, the Taylor-Young expansion around $\gamma=0$ of the permeability (Eq.(24)) is also plotted as a dotted-line curve in Fig.6. Up to a strain of $\gamma=0.2$, the expansion to a quadratic curve, is in good agreement with the permeability calculated with both Eq.(21) and Eq.(22). At higher strain however, the expansion becomes unable to fit the permeability calculated with Eq.(24) because it has reached the linear zone (pre-saturation), as seen in Fig.6.

Thus, for low shear, we can use the Taylor-Young expansion around $\gamma=0$ (Eq.25) to estimate the change of induction:

$$
\Delta B(\gamma \approx 0) \approx \mu_{z}(\gamma) H_{0}-\mu_{z}(\gamma=0) H_{0}=-\mu_{0} b \gamma^{2} H_{0}=-b \gamma^{2} B_{0}
$$

Hence, Eq.(26) can explain the experimental quadratic behavior of the change of induction with the strain observed in Fig.3. On the other hand, Eq.(26) shows a linear dependency on the applied field $B_{0}$ and therefore cannot explain the experimental change of induction with the applied field at the origin of an optimal value. However, such a linear tendency can be attributed to pre-saturation in the magnetic response of the particles.

The dependence of the change of permeability $\Delta \mu_{r}$ on the inter-particle distance and on the filling factor is plotted versus applied strain in Fig.7. Values were calculated from Eq.(21) and (22). Clearly, the change of permeability presented in Fig.7 increases by increasing the filling factor and reducing the inter-particle distance. The model leads to relative permeability changes of about $10^{-1}$ as seen in Fig.7, and in turn, to the change of induction: as for example, at a typical applied field of $10^{-1} \mathrm{~T}, \Delta B \sim 10^{-2} \mathrm{~T}$.
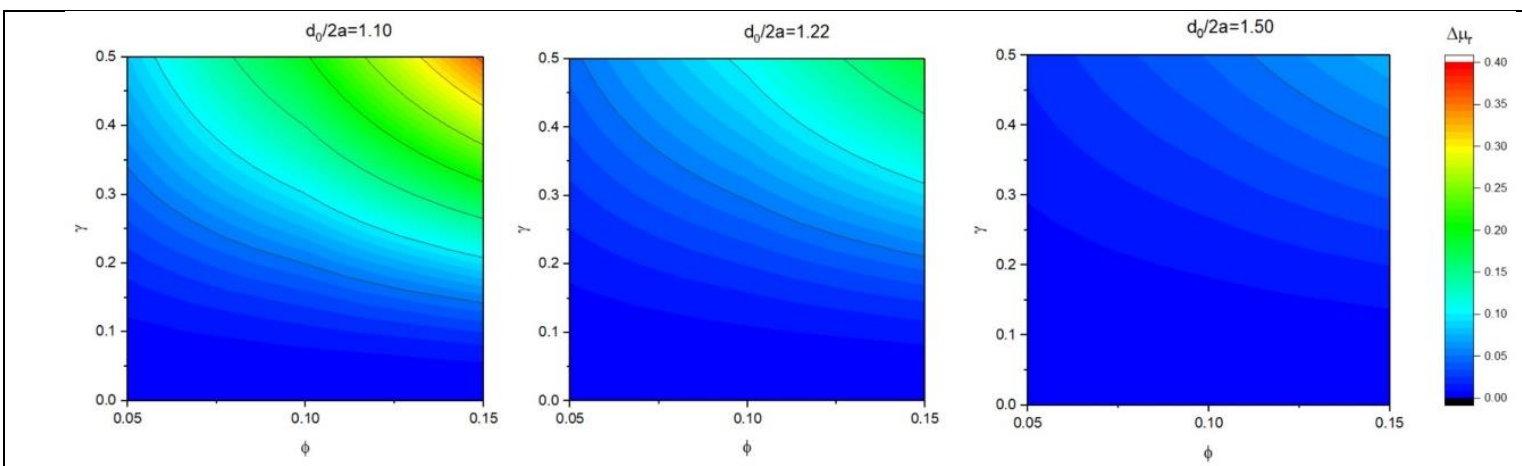

Fig.7. Relative permeability change $\Delta \mu_{r}$ with strain for different filling factor and inter-particle distance 
IV- Non-linear magnetization of a particles chain with saturation

The model detailed in section III can describe the change of induction under shear strain but failed to describe the applied field effect properly at medium and high magnetic fields. In fact, this analytical model assumes a linear behavior between $B$ and $H$ which is incorrect if the applied field is large enough, because magnetic materials always are limited by their magnetic saturation. The permeabilities of these composites exhibit a change of their behavior for field $H$ larger than $100 \mathrm{kA} / \mathrm{m}\left(B_{0} \geq 0.1 \mathrm{~T}\right)$, where the slope is clearly close to 1 (see Fig.2a). This can be understood by writing the susceptibility as:

$\mu=\frac{d B}{d H}=\mu_{0}\left(1+\frac{d M}{d H}\right)$

This equation is similar to Eq.(22) but takes into account the nonlinear effect due to the magnetic saturation of the particles. It appears clearer that the magnetic saturation yields a relative susceptibility of 1 , as $d M / d H$ tends to zero at high $H$. To understand where the nonlinear effect arises, we first estimated the field that saturates the magnetic particles.

The complete magnetization is calculated for a single sphere placed in an external magnetic field $\boldsymbol{H}_{\boldsymbol{O}}$ varying from 0 to around $1000 \mathrm{kA} / \mathrm{m}(\sim 1.26 \mathrm{~T})$ using COMSOL software. For a linear soft ferromagnetic $\left(\mu_{p}=1000 \mu_{0}\right)$ sphere, this isolated sphere is magnetized according to Eq.(4) leading to $\boldsymbol{M}_{p}=3 \boldsymbol{H}_{\boldsymbol{o}}$ by setting $\beta=1\left(\right.$ as $\left.\mu_{p} \gg \mu_{m}\right)$ as represented by dotted line in Fig.8. This is generalized with the demagnetizing factor of the particle as $\boldsymbol{M}_{p}=(1 / N) \boldsymbol{H}_{0}$ where $N=1 / 3$ for a spherical particle. For a nonlinear soft ferromagnetic spherical particle, at low applied field $\boldsymbol{H}_{\boldsymbol{o}}$, such isolated sphere will follow $\boldsymbol{M}_{p}=3 \boldsymbol{H}_{\boldsymbol{0}}$. For example, Iron is selected as the material and the curve is plotted in Fig.8. This Fe particle, at stronger field, reached the saturation of $\boldsymbol{M}_{\text {sat } \_p}=1700 \mathrm{kA} / \mathrm{m}\left(\mu_{0} \boldsymbol{M}_{\text {sat } \_p} \sim 2.14 \mathrm{~T}\right.$ for Fe $)$. The notion of 'low applied field' can be defined as the region where the material has not reached its magnetic saturation. Defining the saturating field $\boldsymbol{H}_{0_{-} \text {sat_p }}$ as the field that brings these particles into the saturation state, this can be estimated by $\boldsymbol{H}_{0_{-} \text {sat_p }}=\boldsymbol{M}_{\text {sat } \_} / 3$, which corresponds to $\boldsymbol{H}_{0_{-} \text {sat_p }} \sim 550 \mathrm{kA} / \mathrm{m}\left(\mu_{0} \boldsymbol{H}_{\boldsymbol{O}_{-} \text {sat } \_p} \sim 0.7 \mathrm{~T}\right)$ for Fe.

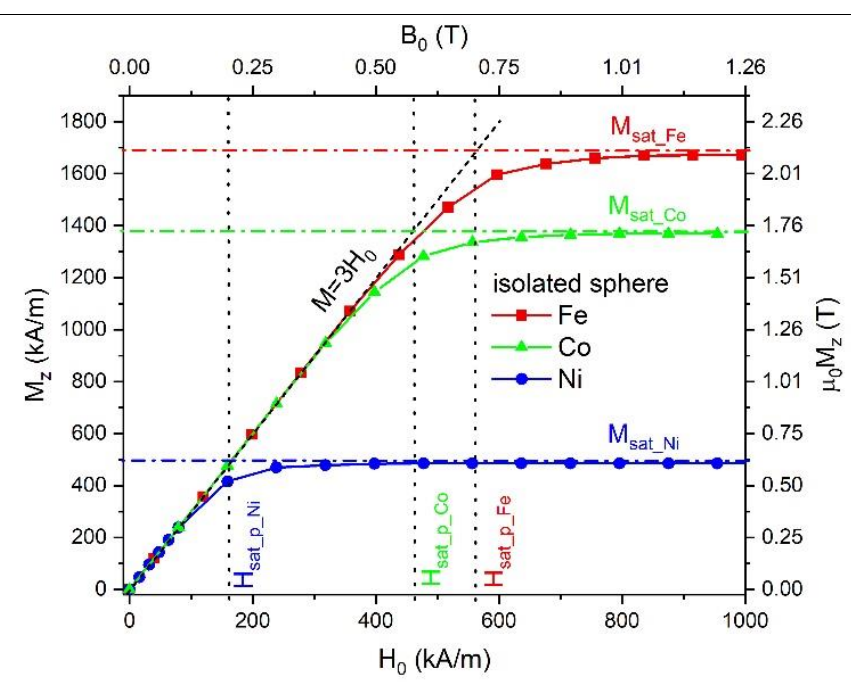

Fig.8. Single sphere magnetization versus applied field calculated using COMSOL for different soft ferromagnetic materials. 
A single Fe particle exhibits a saturating field around $550 \mathrm{kA} / \mathrm{m}$ whereas for the anisotropic composite based on Fe particles, the measured value is assumed to be $100 \mathrm{kA} / \mathrm{m}$ (Fig.2). For an isolated particle, its magnetic behavior is $\boldsymbol{M}=3 \boldsymbol{H}_{\boldsymbol{0}}$ whereas for the composite materials, $\boldsymbol{M}=3 \phi /[1-(\phi+2 D)] \boldsymbol{H}_{0}$ as discussed above. The saturation state of a composite is simply $\boldsymbol{M}=\phi \boldsymbol{M}_{\text {sat } p}$ and does not depend on its structure [20]. The field $\boldsymbol{H}_{0_{-} \text {sat }}$ that brings the composite into the saturation state $\boldsymbol{M}=\phi \boldsymbol{M}_{\text {sat }} \boldsymbol{p}$ is then estimated as: $\boldsymbol{H}_{0_{-} \text {sat }}$ $=\phi \boldsymbol{M}_{\text {sat_p }} /\{3 \phi /[1-(\phi+2 D)]\}$ or $\boldsymbol{H}_{0_{-} \text {sat }}=\boldsymbol{H}_{0_{-} \text {sat } p}[1-\phi-2 D]$, with $\boldsymbol{H}_{0_{-} \text {sat_p }}=\boldsymbol{M}_{\text {sat_p }} / 3$. If the composite is randomly filled $(D=0)$, the saturating field linearly decreases at increasing particle volume fraction as $\boldsymbol{H}_{0_{-} \text {sat }}=\boldsymbol{M}_{\text {sat } \_p}(1-\phi) / 3$ [21]. The presence of other particles is reducing the saturating field of an isotropic composite to a weaker value than the saturating field of single particle. Then, if the composite is not randomly-filled $(D \neq 0)$, the expression $\boldsymbol{H}_{0_{-} \text {sat }}=\boldsymbol{H}_{0_{-} \text {sat_p }}[1-\phi-2 D]$ can help in understanding that for a given filling factor $\phi$, an anisotropic composite has a different saturating field than an isotropic composite. According to the previous calculations, for an applied field collinear to the particle column, $2 D=4 \mathrm{~S}\left(\mathrm{a} / \mathrm{d}_{0}\right)^{3}$, then $D>0$, and this anisotropic composite has a lower saturating field than an isotropic composite, as seen in Fig.2a.

However, this simple expression is limited to diluted composites but it shows that a saturation at field of $\boldsymbol{H}_{0_{-} \text {sat }} \sim 100 \mathrm{kA} / \mathrm{m}$, as measured in Fig.2, can be naturally found in composite with column of particles when the magnetization is measured along this anisotropy axis.

Using COMSOL software, a model was constructed with five ferromagnetic particles, with nonlinear magnetic behavior included. With five particles, the sum (Eq.8) is S 1.185 close to the exact value (1.202) so that the modelling is a good compromise between simplicity and reality. They were placed at different positions in the z-y plan as in Fig. 4 to simulate a pure shear process. A representative dilute composite cell was created by diluting these particles within a non-magnetic volume. In this cell, the single chain is composed of 5 particles with initial inter-particle distance $d / 2 a=1.1$ (and $d_{y}=0$, see Fig.4). At increasing shear deformation, $d_{z} / 2 a$ remains constant at 1.1 , while $d_{y}$ increases. The magnetic induction, magnetic field and magnetization are averaged inside the cell. The magnetic induction change is plotted versus the particle displacement $d_{y}$ in Fig.9a and versus applied field in Fig.9b. This magnetic induction $\Delta B_{z}$ change has a quadratic behavior at low $d_{y}$ then seems to be linear. This is similar to the composite permeability plotted in Fig3, Fig5 and Fig6. On the other hand, as the magnetization versus the applied field $\boldsymbol{H}_{\boldsymbol{0}}$, (and thus $\boldsymbol{B}_{0}$ ), is not a linear function as described in Eq.(4), the curve amplitudes are first increasing with $\boldsymbol{B}_{\boldsymbol{0}}$ from 0.1 to $0.3 \mathrm{~T}$, and then are decreasing to lower values at stronger $\boldsymbol{B}_{\boldsymbol{0}}$.

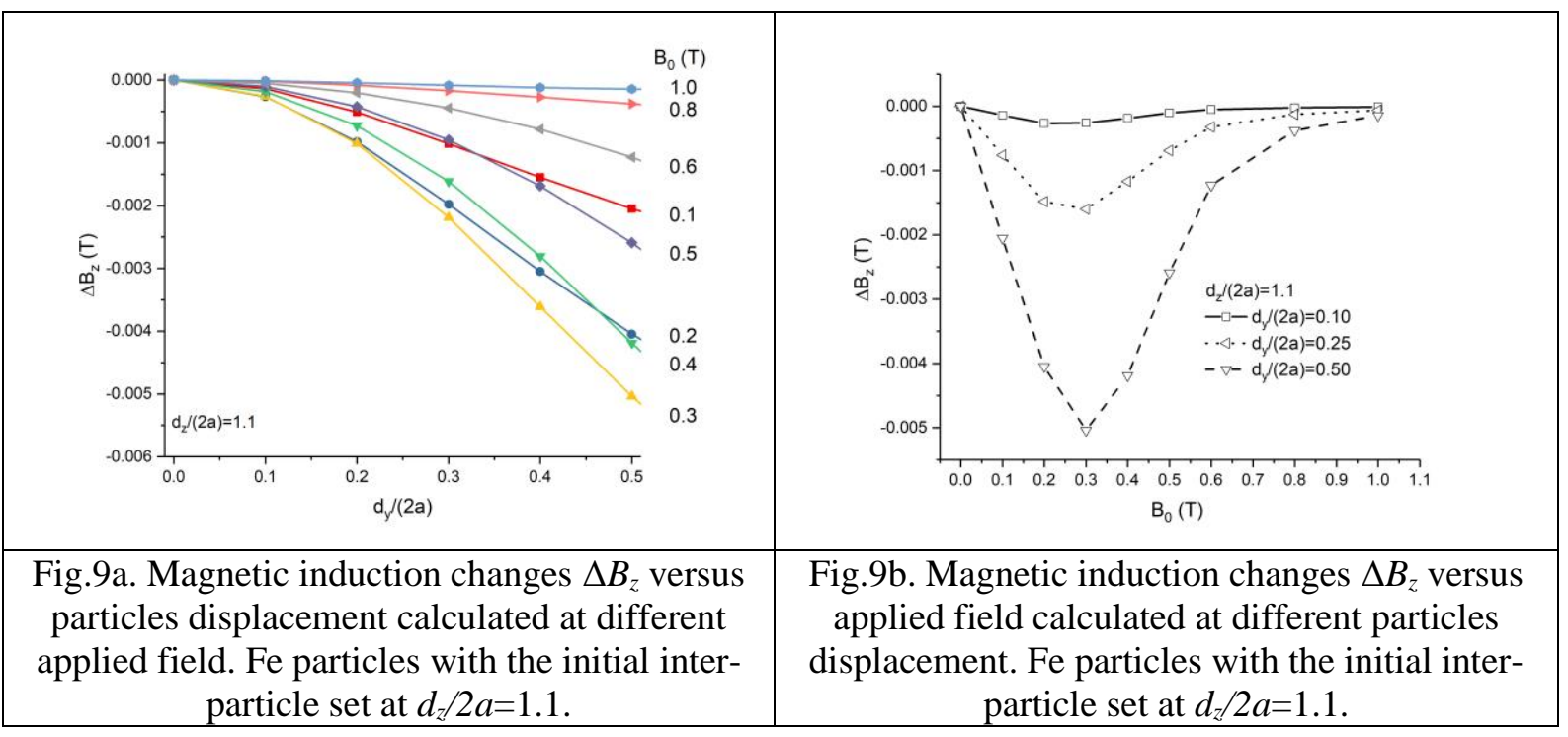


The corresponding magnetization curves at $d_{z} / 2 a=1.1$ are plotted in Fig.10a under shear strain $\left(d_{y} / 2 a=\right.$ 0.5 ) and at without shear strain $\left(d_{y} / 2 a=0\right)$. In both cases, they reach the same saturation state (for Fe: $\left.\phi \boldsymbol{M}_{p_{-} s a t}=37.5 \mathrm{kA} / \mathrm{m}\right)$, but the curve with displaced particles has a weaker initial slope as discussed in the linear model section (section III). Consequently, the saturation field without displacement $\left(d_{y} / 2 a=0\right)$ is weaker than with displacement $\left(d_{y} / 2 a=0.5\right)$; extracted values from Fig.10a for Fe are $\boldsymbol{H}_{\boldsymbol{0}_{-} \text {sat }} \sim 345 \mathrm{kA} / \mathrm{m}$ $(0.43 \mathrm{~T})$ and $430 \mathrm{kA} / \mathrm{m}(0.54 \mathrm{~T})$ respectively.

The change of induction $\Delta B_{z}$ is plotted in Fig.10b for Fe with different initial $d_{z} / 2 a=1.1,1.2,1.5$ and 1.7. The change of induction obtained with $d_{z} / 2 a=1.1$ shows an optimal applied field at $0.3 \mathrm{~T}$ as already mentioned from Fig.9b and similarly to the change of magnetization, Fig.10a. More generally, these two curves have the same behavior. As a matter of fact, the change of induction is proportional to the change of magnetization of this composite with a single chain. This comes from the fact that the induction variation is simply the sum of the magnetic field and the magnetization, where the magnetization of the particle is changing with particle displacement. Only the magnetic field created by the particles (Eq.10) varies (the applied field remains constant).

Finally, we compare the state of magnetization of the particles in the composite; we set two other nonlinear materials beside Fe and the linear material (with $\mu_{\mathrm{r}}=1000$ ). Selected ferromagnetic materials were Nickel and Cobalt. At low field, the magnetization of all materials follows $\boldsymbol{M}_{p}=3 \boldsymbol{H}_{\boldsymbol{0}}$ because it only depends on particle shape. The magnetic saturations are $1370 \mathrm{kA} / \mathrm{m}$ for Co and $485 \mathrm{kA} / \mathrm{m}$ for Ni as seen in Fig.8, which corresponds to $\mu_{0} \boldsymbol{M}_{\text {sat } \_p} \sim 1.72 \mathrm{~T}$ for Co and $0.61 \mathrm{~T}$ for Ni. Saturating fields of single particles $\left(\boldsymbol{H}_{\boldsymbol{O}_{\_} \text {sat } \_}\right)$are then estimated as $456 \mathrm{kA} / \mathrm{m}\left(\mu_{0} \boldsymbol{H}_{0_{-} \text {sat_p }} \sim 0.57 \mathrm{~T}\right)$ for Co and $160 \mathrm{kA} / \mathrm{m}\left(\mu_{0} \boldsymbol{H}_{\boldsymbol{0}_{-} \text {sat_p }}\right.$ $\sim 0.2 \mathrm{~T}$ ) for Ni.

Strain effect on magnetization curves are plotted in Fig.10a with $d_{y} / 2 a=0.5$ and $d_{y} / 2 a=0$, as well as with the corresponding change of magnetization. Unsurprisingly, the linear material does not present any saturation. Hence, its change of magnetization $\Delta \boldsymbol{M}$ is monotonously varying. On the other hand, $\mathrm{Fe}$, $\mathrm{Ni}$ and $\mathrm{Co}$ are presenting some saturation and their change of magnetization $\Delta \boldsymbol{M}$ are first increasing before coming back to zero after saturation. It can be observed that the largest variation of magnetization due to the applied strain, at constant applied field $|\Delta M|$ max increases with the material magnetic saturation $(|\Delta \boldsymbol{M}| \max =4.34 \mathrm{kA} / \mathrm{m}$ for $\mathrm{Fe}, 1.12 \mathrm{kA} / \mathrm{m}$ for Co and $3.48 \mathrm{kA} / \mathrm{m}$ for $\mathrm{Ni})$. Consequently, the same behavior is observed for $|\Delta \boldsymbol{B}| \max$ as seen in Fig.10b. Clearly, the magnetic state of the particles inside the composite is a key parameter in energy harvesting systems based on shear strain of MREs.

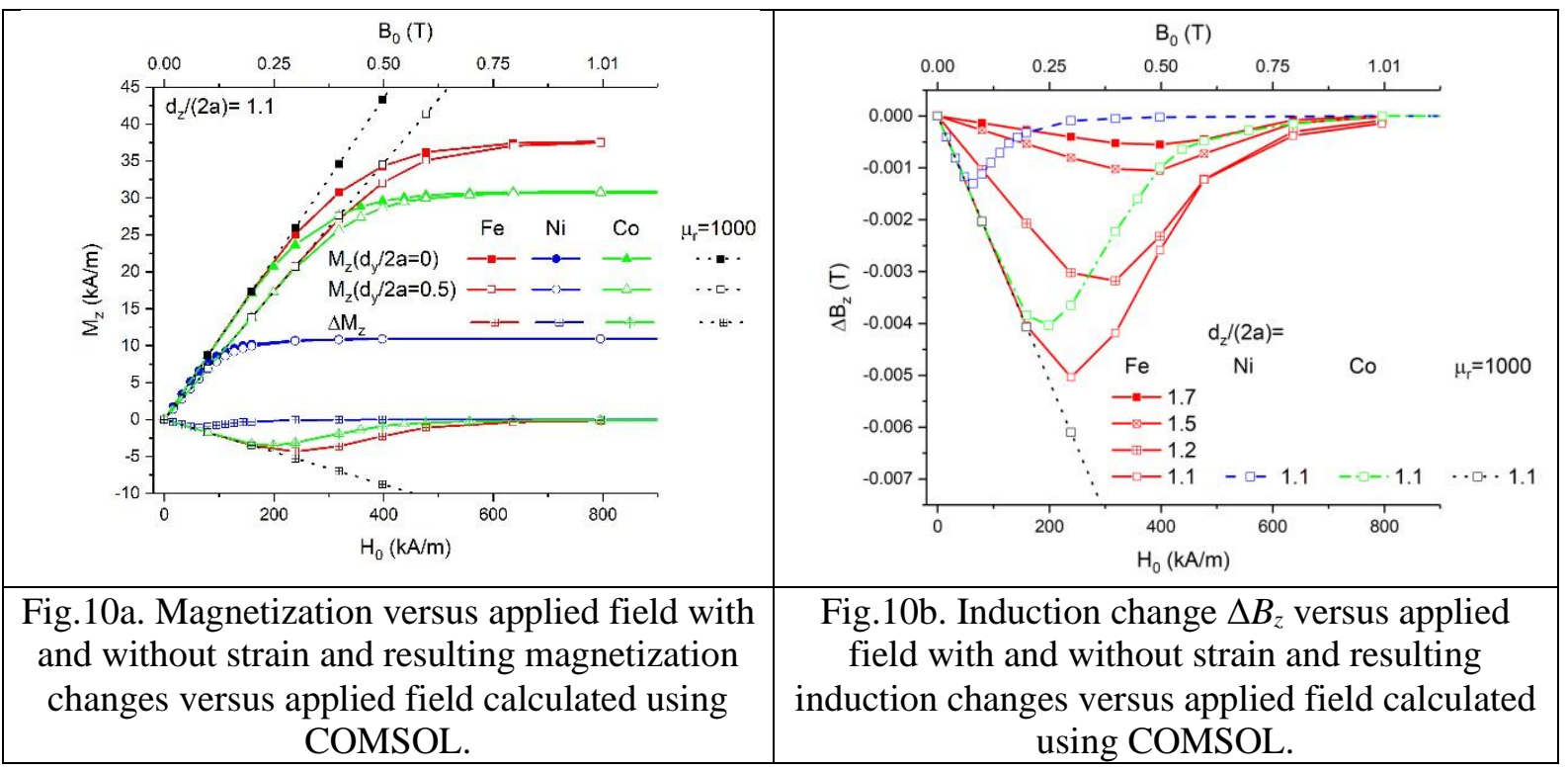


The change of magnetic induction of a MRE submitted to a transverse shear presents an optimal value at a given applied magnetic field. The applied shear is changing the magnetic state inside the magnetic materials. For MRE, it is usually the magnetic particles that is mainly the magnetic material, the elastomer is easing the movement of these particles when the MRE is submitted to a mechanical stress. In this paper, we calculated the change of magnetization of a particle inside a single chain of magnetic particles embedded in a non-magnetic material, submitted to a pure shear strain. Results show that particles aligned with the external applied field have their magnetization larger than misaligned particles because the field induced by the particles is lower in the latter case. Two contributions lead to this result, one is purely due to the angle of the chain with the applied field and the second one is due to the interdistance which is increased by the shear strain. The change of the particles magnetization in the chain results in a change of the magnetic induction of the whole composite in which the chain is embedded.

We demonstrated the effect of the magnetic saturation which leads to a decrease of the particle magnetization change. In this way, the change of the magnetic induction of a composite is also altered as the applied field approaches the saturating field of the particle. Simple expression is provided here to estimate the value of the saturating field of particle. Moreover, the ratio between the particle size and inter particle distance ( $a / d_{z}$ from Eq.9) is tuning the effect as seen in Fig $10 b$; this agrees with prediction from the model of Ginger [22] for the elastic modulus change $\Delta \mathrm{G}=\phi \mathrm{M}_{\mathrm{s}}{ }^{2} /\left[2 \mu_{\mathrm{m}}\left(\mathrm{d}_{\mathrm{z}} / \mathrm{a}\right)^{3}\right]$. The effect of the distribution of particle size or inter particle distance can be estimated by averaging the $d_{z} / 2 a$ ratio in Fig.10b; the resulting curve would have same shape but with an intermediate peak value.

For a more accurate modelling, especially with larger filling factor, where the chains are not anymore isolated, other contributions have to be taken into account. These contributions require more information on the microstructure, such as the distance between neighboring particles in a chain and the distance between neighboring chains. Such parameters are known to depend on the filling factor, and moreover on the curing condition like applied field, temperature, anti-sedimentation process (spinning of the sample), etc. As mentioned in section IV, the magnetization depends on the demagnetizing factor $N$ as $\boldsymbol{M}_{p}=(1 / N) \boldsymbol{H}_{\boldsymbol{0}}$ implying that the particle shape should also plays a role and, especially as a magnetomechanical effect. Generally speaking, the magneto-mechanical effects are dynamics properties where parameters such as the strain rate might be important but, this can be discussed in further work.

Acknowledgement

References:

The work has been performed in the frame of the Japan - France International Laboratory (LIA) ELyTGlobal. The authors gratefully acknowledge for their support the French Region Auvergne-RhôneAlpes, and support from French project IDEXLYON of the Université de Lyon in the frame of the "Investissements d'Avenir" program (ANR-16-IDEX-0005). 
[2] Magnetic field sensitive functional elastomers with tuneable elastic modulus, Z. Varga, G. Filipcsei, M. Zrínyi, Polymer (2006) 47 227-233. https://doi.org/10.1016/j.polymer.2005.10.139

[3] From dipolar interactions of a random distribution of ferromagnetic particles to magnetostriction, $G$. Diguet, E. Beaugnon, J.Y. Cavaillé, J. Mag. Mag. Mater. (2009) 321 396-401. https://doi.org/10.1016/j.jmmm.2008.08.112

[4] Shape effect in the magnetostriction of ferromagnetic composite, G. Diguet, E. Beaugnon, J.Y. Cavaillé, J. Mag. Mag. Mater. (2010) 322 3337-3341. https://doi.org/10.1016/j.jmmm.2010.06.020

[5] Dependence of the magnetostriction of magnetic rheological elastomers on temperature, G. Diguet, E. Beaugnon, J.Y. Cavaillé, Smart Mater. Struct. (2012) 21 025016. https://doi.org/10.1088/0964$1726 / 21 / 2 / 025016$

[6] The Giant linear magnetostriction in elastic ferromagnetic composites within a porous matrix, S. Bednarek, J. Mag. Mag. Mater. (2006) 301 200-207. https://doi.org/10.1016/j.jmmm.2005.05.041

[7] Deformation in magnetorheological elastomer and elastomer-ferromagnet composite driven by a magnetic field, G.Y. Zhou, Z.Y. Jiang, Smart Mater. Struct. (2004) 13 309-316. https://doi.org/10.1088/0964-1726/13/2/009

[8] Magnetostriction of field-structured magnetoelastomers, J.E. Martin, R.A. Anderson, D. Read, G. Gulley, Phys. Rev. E (2006) 74 051507. https://doi.org/10.1103/PhysRevE.74.051507

[9] Development and characterization of a magnetorheological elastomer based adaptive seismic isolator, Y. Li, J. Li, W. Li, B. Samali, Smart Mater. Struct. (2012) 22035005. https://doi.org/10.1088/0964-1726/22/3/035005

[10] Development of an adaptive tuned vibration absorber with magnetorheological elastomer, H.X. Deng, X.L. Gong, L.H. Wang, Smart Mater. Struct. (2006) 15 N111. https://doi.org/10.1088/0964$1726 / 15 / 5 / \mathrm{N} 02$

[11] Magnetic Field Sensitive Polymeric Actuators, M. Zrinyi, D. Szabo, L. Barsi, J. intell. Mater. Syst. Struct. (1998) 9 667-671. https://doi.org/10.1177/1045389X9800900814

[12] State of the art and development trends of novel nanostructured elastomagnetic composites, L. Lanotte, G. Ausanio, C. Hison, V. Iannotti, C. Luponio, C. Luponio Jr., J. Optoelectron. Adv. Mater. (2004) 6 523-532.

[13] Anisotropic magnetorheological elastomers for mechanical to electrical energy conversion, $\mathrm{M}$. Lallart, G. Sebald, G. Diguet, J.Y. Cavaille, M. Nakano, J. Appl. Phys. (2017) 122103902. https://doi.org/10.1063/1.4998999

[14] Energy conversion in magneto-rheological elastomers, G. Sebald, M. Nakano, M. Lallart, T. Tian, G. Diguet J.Y. Cavaille, Science Tech. Adv. Mater. (2017) 18 766-778. https://doi.org/10.1080/14686996.2017.1377590

[15] Optimal design of MR shock absorber and application to vehicle suspension, Q.H. Nguyen, S.B. Choi, Smart Mater. Struct. (2009) 18 035012. https://doi.org/10.1088/0964-1726/18/3/035012

[16] Optimal design of MR damper via finite element analyses of fluid dynamic and magnetic field, Z. $\begin{array}{llllll}\text { Parlak, T. Engin, I Çalli, Mechatronics (2012) } 22 & \text { 890-903. }\end{array}$ https://doi.org/10.1016/j.mechatronics.2012.05.007

[17] Anisotropic magnetism in field-structured composites, J.E. Martin, E. Venturini, J. Odinek, R.A. Anderson, Phys. Rev. E (2000) 61 2818. https://doi.org/10.1103/PhysRevE.61.2818 
1 [18] Microstructure-property relationships of urethane magnetorheological elastomers, A. Boczkowska, 2 S.F. Awietjan, R. Wroblewski, Smart Mater. Struct. (2007) 16 1924. https://doi.org/10.1088/0964$3 \quad 1726 / 16 / 5 / 049$

4 [19] Image analysis of the microstructure of magnetorheological elastomers, A. Boczkowska, S.F. 5 Awietjan, T. Wejrzanowski, J. Mater. Science (2009) 44 31353140. https://doi.org/10.1007

6 [20] Huge Magnetostriction of Magneto-rheological composite, G. Diguet, thesis (2010). 7 https://tel.archives-ouvertes.fr/tel-00488910/. Fig.IV.18

8 [21] Dynamic shear response of hard versus soft magnetic magnetoactive elastomers, K. Anderson, R. 9 Bravoco, W. Hargrave, J. Roche, P. Von Lockette, S.E. Lofland, Smart Mater. Struct. (2015) 24025022. 10 https://doi.org/10.1088/0964-1726/24/2/025022

11 [22] A model of the behavior of magnetorheological materials, M.R. Jolly, J.D. David, B.C. Munoz, Smart Mater. Struct. (1996) 5 607-614. https://doi.org/10.1088/0964-1726/5/5/009 
2 Appendix A

3 Starting from the following definitions:

$4 \tan (\theta)=\frac{d_{y}}{d_{0}}=\gamma$

$5 \quad \sin (\theta)=\frac{d_{y}}{d}$

$6 \cos (\theta)=\frac{d_{0}}{d}$

7 Then, by using $d^{2}=d_{\mathrm{y}}^{2}+d_{0}^{2}$, we can get the following expressions:

$8 \sin ^{2}(\theta)=\frac{d_{y}^{2}}{d_{y}^{2}+d_{0}^{2}}=\frac{\gamma^{2} d_{0}^{2}}{\gamma^{2} d_{0}^{2}+d_{0}^{2}}=\frac{\gamma^{2}}{\gamma^{2}+1}$

$9 \cos (\theta) \sin (\theta)=\frac{d_{0} d_{y}}{d_{y}^{2}+d_{0}^{2}}=\frac{d_{0} \gamma d_{0}}{\gamma^{2} d_{0}^{2}+d_{0}^{2}}=\frac{\gamma}{\gamma^{2}+1}$

$10 \quad d^{3}=\left(d_{y}^{2}+d_{0}^{2}\right)^{3 / 2}=\left(\gamma^{2} d_{0}^{2}+d_{0}^{2}\right)^{3 / 2}=d_{0}^{3}\left(\gamma^{2}+1\right)^{3 / 2}$

11

12

14

$$
A(\gamma, \delta)=\frac{3 \phi \beta}{1-\beta\left(\phi+\delta S \frac{a^{3}}{d_{0}^{3}} \frac{1}{\left(\gamma^{2}+1\right)^{3 / 2}}\right)}
$$

The main difference between the functions $\alpha$ and $A$ being the filling factor parameter $\phi$ is inserted for taking into account the composite field.

17 The permeability expressed in Eq.(21) and Eq.(22) can be rewritten as:

18

$\mu_{z}=\mu_{0}\left(1+A(\gamma, 4)-\frac{\gamma^{2}}{\gamma^{2}+1}[A(\gamma, 4)-A(\gamma,-2)]\right)$

19

The Taylor-Young expansion around $\gamma=0$ for low shear, Eq.(23), can be then estimated from the Eq.(B.2) and Eq.(B.1)

\footnotetext{
First, we need to differentiate $A$ with respect to the strain:
} 
$1 \frac{\partial A(\gamma, \delta)}{\partial \gamma}=-\frac{3 \phi \beta}{\left(1-\beta\left(\phi+\delta S \frac{a^{3}}{d_{0}^{3}} \frac{1}{\left(\gamma^{2}+1\right)^{3 / 2}}\right)\right)^{2}} \frac{\left(-\beta \delta S \frac{a^{3}}{d_{0}^{3}}\right)\left(-\frac{3}{2} 2 \gamma\right)}{\left(\gamma^{2}+1\right)^{5 / 2}}$

2 Or more simple as:

$3 \quad \frac{\partial A(\gamma, \delta)}{\partial \gamma}=-\delta S \frac{a^{3}}{d_{0}^{3}} \frac{A^{2}(\gamma, \delta)}{\phi} \frac{\gamma}{\left(\gamma^{2}+1\right)^{5 / 2}}$

4 Then, we need to differentiate Eq.(B.4) to obtain the second derivative of $A$ as:

$5 \quad \frac{\partial^{2} A(\gamma, \delta)}{\partial \gamma^{2}}=-\delta S \frac{a^{3}}{d_{0}^{3}} \frac{A(\gamma, \delta)}{\phi} \frac{1}{\left(\gamma^{2}+1\right)^{5 / 2}}\left(2 \frac{\partial A(\gamma, \delta)}{\partial \gamma} \gamma+A(\gamma, \delta)\left(1-\frac{5 \gamma^{2}}{\left(\gamma^{2}+1\right)}\right)\right)$

6 The first derivative of Eq.(B.2) is then expressed using the function $A$ with Eq.(B.4) as:

$7 \quad \frac{\partial \mu_{z}}{\partial \gamma}=\mu_{0}\left(\frac{\partial A(\gamma, 4)}{\partial \gamma}-\frac{\gamma^{2}}{\gamma^{2}+1}\left[\frac{\partial A(\gamma, 4)}{\partial \gamma}-\frac{\partial A(\gamma,-2)}{\partial \gamma}\right]-\frac{2 \gamma}{\left(\gamma^{2}+1\right)^{2}}[A(\gamma, 4)-A(\gamma,-2)]\right)$ (B.6)

8 It is worthy to notice that the value at $\gamma=0$ is null since:

$9 \quad \frac{\partial \mu_{z}(\gamma=0)}{\partial \gamma}=\mu_{0}\left(\frac{\partial A(0,4)}{\partial \gamma}\right)=0$

10

11

12

14

15

16

17

The second derivative of Eq.(B.2) is then expressed using the function $A$ with Eq.(B.4) and Eq.(B.6) as:

$\frac{\partial^{2} \mu_{z}}{\partial \gamma^{2}}=\mu_{0}\left(\frac{\partial^{2} A(\gamma, 4)}{\partial \gamma^{2}}-\frac{\gamma^{2}}{\gamma^{2}+1}\left[\frac{\partial^{2} A(\gamma, 4)}{\partial \gamma^{2}}-\frac{\partial^{2} A(\gamma,-2)}{\partial \gamma^{2}}\right]-\frac{2 \gamma}{\left(\gamma^{2}+1\right)^{2}}\left[\frac{\partial A(\gamma, 4)}{\partial \gamma}-\frac{\partial A(\gamma,-2)}{\partial \gamma}\right]-2 \frac{1-3 \gamma^{2}}{\left(\gamma^{2}+1\right)^{3}}[A(\gamma, 4)-A(\gamma,-2)]-\frac{2 \gamma}{\left(\gamma^{2}+1\right)^{2}}\left[\frac{\partial A(\gamma, 4)}{\partial \gamma}-\frac{\partial A(\gamma,-2)}{\partial \gamma}\right]\right)$

(B.8)

The second derivative value at $\gamma=0$ is:

$\frac{\partial^{2} \mu_{z}(\gamma=0)}{\partial \gamma^{2}}=\mu_{0}\left(\frac{\partial^{2} A(0,4)}{\partial \gamma^{2}}-2[A(0,4)-A(0,-2)]\right)$

The combination of Eq.(B.2), Eq.(B.7) and Eq.(B.9) with The Taylor-Young expansion around $\gamma=0$ for low shear, Eq.(23), is providing the low shear permeability equation as:

$\mu_{z}(\gamma=0) \approx \mu_{0}\left[1+A(0,4)+\frac{\gamma^{2}}{2}\left(\frac{\partial^{2} A(0,4)}{\partial \gamma^{2}}-2[A(0,4)-A(0,-2)]\right)\right]+\ldots$

And it final form is expressed by injecting Eq.(B.1) and Eq.(B.5), as:

$\mu_{z}(\gamma=0) \approx \mu_{0}\left[1+\frac{3 \phi \beta}{1-\beta\left(\phi+4 S \frac{a^{3}}{d_{0}^{3}}\right)}+\frac{\gamma^{2}}{2}\left(-4 S \frac{a^{3}}{d_{0}^{3}} \frac{A^{2}(0,4)}{\phi}-2 \frac{3 \phi \beta}{1-\beta\left(\phi+4 S \frac{a^{3}}{d_{0}^{3}}\right)}+2 \frac{3 \phi \beta}{1-\beta\left(\phi-2 S \frac{a^{3}}{d_{0}^{3}}\right)}\right)\right]+\ldots$ 
1 And by injecting $2 D=4 \mathrm{~S}\left(\mathrm{a} / \mathrm{d}_{0}\right)^{3}$,

$2 \quad \mu_{z}(\gamma=0) \approx \mu_{0}\left[1+\frac{3 \phi \beta}{1-\beta(\phi+2 D)}-\gamma^{2}\left(D \frac{9 \phi \beta^{2}}{(1-\beta(\phi+2 D))^{2}}+\frac{3 \phi \beta}{1-\beta(\phi+2 D)}-\frac{3 \phi \beta}{1-\beta(\phi-D)}\right)\right]+\ldots$ (B.12)

3

4

5

6 